\title{
Reflection equation algebra in braided geometry ${ }^{1}$
}

\author{
Dimitri GUREVICH ${ }^{a}$, Pavel PYATOV ${ }^{b}$, and Pavel SAPONOV ${ }^{c}$ \\ ${ }^{a}$ USTV, Université de Valenciennes, 59313 Valenciennes, France \\ E-mail: gurevich@univ-valenciennes.fr \\ ${ }^{b}$ Bogoliubov Laboratory of Theoretical Physics, JINR, 141980 Dubna, Moscow region, Russia \\ E-mail: pyatov@theor.jinr.ru \\ ${ }^{c}$ Division of Theoretical Physics, IHEP, 142281 Protvino, Moscow region, Russia \\ E-mail: Pavel.Saponov@ihep.ru
}

\begin{abstract}
Braided geometry is a sort of the noncommutative geometry related to a braiding. The central role in this geometry is played by the reflection equation algebra associated with a braiding of the Hecke type. Using this algebra, we introduce braided versions of the Lie algebras $g l(n)$ and $\operatorname{sl}(n)$. We further define braided analogs of the coadjoint orbits and the vector fields on a $q$-hyperboloid which is the simplest example of a "braided orbit". Besides, we present a braided version of the Cayley-Hamilton identity generalizing the result of Kantor and Trishin on the super-matrix characteristic identities.
\end{abstract}

2000 MSC: 17B37, 81R50

Key words: (modified) reflection equation algebra, braiding, Hecke symmetry, HilbertPoincaré series, bi-rank, Cayley-Hamilton identity, $q$-hyperboloid, braided vector fields

In memory of our friend Issai Kantor

\section{Introduction}

By braided geometry we understand a sort of the noncommutative geometry related to a braiding $R$, which is an invertible operator $R: V^{\otimes 2} \rightarrow V^{\otimes 2}$ obeying to the Yang-Baxter equation

$$
(R \otimes I)(I \otimes R)(R \otimes I)=(I \otimes R)(R \otimes I)(I \otimes R)
$$

Here $V$ is a vector space over the ground field $\mathbb{K}=\mathbb{C}$ (or $\mathbb{R}$ ).

In what follows we are dealing with braidings satisfying a second order equality

$$
(q I-R)\left(q^{-1} I+R\right)=0
$$

where $q \in \mathbb{K}$ is assumed to be generic (in particular, this means $q^{k} \neq 1$ for any integer $k \geq 2$ ). Such a braiding is called a Hecke symmetry. It becomes an involutive symmetry in case $q=1$.

Let $U_{q}(s l(n)) \rightarrow \operatorname{End}(\mathrm{V})$ be the basic representation of the quantum group $U_{q}(s l(n)), \operatorname{dim} \mathrm{V}=$ n. Then the product of the image of the quantum universal $R$-matrix in the space $V^{\otimes 2}$ and the flip (transposition operator) is a Hecke symmetry. In what follows this Hecke symmetry will be referred to as a standard one. Note that its limit at $q \rightarrow 1$ is the usual flip. In this sense we can treat the standard Hecke symmetry as a deformation of the flip. In a similar way one can construct deformation of a super-flip. Besides, there are known Hecke symmetries which are neither deformations of a flip, nor of a super-flip (see the next section).

\footnotetext{
${ }^{1}$ Presented at the $3^{\text {rd }}$ Baltic-Nordic Workshop "Algebra, Geometry, and Mathematical Physics ", Göteborg, Sweden, October 11-13, 2007.
} 
With any Hecke symmetry we can associate (at least) two matrix algebras. One of them is called RTT (or Reshetikhin-Takhtajan-Faddeev) algebra (cf [17]), the other one is called Reflection Equation Algebra (REA) ${ }^{2}$.

Both, the RTT and the RE algebras (supplied with a spectral parameter) are employed in constructing integrable dynamical models. Besides, the latter algebra possesses a number of remarkable properties which make it a very interesting object of the braided geometry.

First, the REA admits some quotients which can be regarded as noncommutative (braided) analogs of the coadjoint orbits. Moreover, it enables one to define a braided analog of the Lie bracket.

Second, for certain matrices with entries belonging to REA one can find the Cayley-Hamilton $(\mathrm{CH})$ identities with central coefficients. These identities allow one to define a whole family of projective modules over the braided orbits. An attempt to develop a sort of related K-theory based on a "braided trace" instead of the usual one, which is traditionally employed in the classical K-theory, was made in [8]. Most of these objects are defined and studied for the REA related to the so called even Hecke symmetries (see the next section).

For the general linear type Hecke symmetries (further referred to as the $G L(p \mid r)$ type symmetries) we have established the basic $\mathrm{CH}$ identity. This identity is a $q$-extension of the characteristic identity for super-matrices discovered by I.Kantor and I.Trishin in [13]. Note that it is this remarkable paper which stimulated our interest in the REAs related to the $G L(p \mid r)$ type Hecke symmetries.

The present paper is a brief review of the main properties of the REA and their applications in braided geometry. In particular, we present a classification of the Hecke symmetries (section 2), define and compare the corresponding quantum matrix algebras (section 3), introduce braided analogs of the Lie algebras $g l(n)$ and $\operatorname{sl}(n)$ (section 4), and consider braided version of the CayleyHamilton identity (section 5). We conclude the paper with an example of a $q$-hyperboloid for which we describe a braided analog of vector fields and describe their applications.

\section{Classification of Hecke symmetries}

Definition 2.1. Let $V$ be a finite dimensional vector space over the field $\mathbb{K}, \operatorname{dim}_{\mathbb{K}} V=n$. An invertible operator $R: V^{\otimes 2} \rightarrow V^{\otimes 2}$ is called a braiding if it satisfies the quantum Yang-Baxter equation

$$
(R \otimes I)(I \otimes R)(R \otimes I)=(I \otimes R)(R \otimes I)(I \otimes R)
$$

If, in addition, a braiding $R$ obeys the relation

$$
(q I-R)\left(q^{-1} I+R\right)=0, \quad q \in \mathbb{K} \backslash 0
$$

it is called a Hecke symmetry. If $q=1$ a braiding $R$ is called an involutive symmetry.

For any Hecke symmetry the braided analogs of the symmetric and skew-symmetric algebras of the space $V$ can be constructed in the following way. Define the "R-symmetric" $I_{+}$and "R-skew-symmetric" $I_{-}$subspaces of $V^{\otimes 2}$ by the relations

$$
\begin{aligned}
& I_{+}:=\operatorname{Im}\left(q^{-1} I+R\right) \quad \text { i.e } \quad I_{+}=\left\{q^{-1} x \otimes y+R(x \otimes y), \forall x, y \in V\right\} \\
& I_{-}:=\operatorname{Im}(q I-R) \quad \text { i.e } \quad I_{-}=\{q x \otimes y-R(x \otimes y), \forall x, y \in V\}
\end{aligned}
$$

\footnotetext{
${ }^{2} \mathrm{~A}$ method of constructing other matrix algebras by means of a pair of compatible braidings, one of them being the Hecke symmetry, is described in $[11,5]$.
} 
Let $T(V)$ denotes the free tensor algebra of the space $V$, and $\left\langle I_{ \pm}\right\rangle$stands for a two-sided ideal in $T(V)$ generated by the space $I_{ \pm}$. The quotients

$$
\Lambda_{+}(V):=T(V) /\left\langle I_{-}\right\rangle \quad \text { and } \quad \Lambda_{-}(V):=T(V) /\left\langle I_{+}\right\rangle
$$

are called $R$-symmetric and $R$-skew-symmetric algebras of the space $V$ respectively.

Noticing that $\Lambda_{ \pm}(V)$ are quadratic graded algebras, we consider their Hilbert-Poincaré (HP) series

$$
\mathcal{P}_{+}(t):=\sum_{k \geq 0} \operatorname{dim} \Lambda_{+}^{k}(V) t^{k}, \quad \mathcal{P}_{-}(t):=\sum_{k \geq 0} \operatorname{dim} \Lambda_{-}^{k}(V) t^{k}
$$

where $\Lambda_{ \pm}^{k}(V) \subset \Lambda_{ \pm}(V)$ are homogeneous components of the degree $k$.

Theorem 2.2. For generic values of $q$ the following identity holds

$$
\mathcal{P}_{+}(t) \mathcal{P}_{-}(-t)=1
$$

Example 1. If $R=P$ where $P$ is the usual flip then $\mathcal{P}_{-}(t)=(1+t)^{n}$ where $n=\operatorname{dim} V$.

Example 2. If $V=V_{0} \oplus V_{1}$ is a super-space, $s \operatorname{dim} V=(p \mid r)$ and $R$ is the corresponding super-flip then $\mathcal{P}_{-}(t)=\frac{(1+t)^{p}}{(1-t)^{r}}$.

Definition 2.3. If $\mathcal{P}_{-}(t)$ (respectively $\mathcal{P}_{+}(t)$ ) is a monic polynomial then the space $V$ and the braiding $R$ are called even (respectively odd).

Theorem 2.4. If $R$ is even then the polynomial $\mathcal{P}_{-}(t)$ is reciprocal.

Proposition 2.5. For any $V$ with $\operatorname{dim} V=n \geq 2$ there exists a Hecke symmetry $R: V^{\otimes 2} \rightarrow V^{\otimes 2}$ such that $\mathcal{P}_{-}(t)=1+n t+t^{2}$.

Note that if $n>2$ such a Hecke symmetry cannot be a deformation of the usual flip since the HP series are stable under the deformation.

Definition 2.6. Let $R$ be an even Hecke symmetry. The degree $p=\operatorname{deg} \mathcal{P}_{-}(t)$ is called the rank of $R$.

Proposition 2.7. Let $p$ be an integer constrained by $2 \leq p \leq n=\operatorname{dim} V$. Then there exists an even Hecke symmetry $R: V^{\otimes 2} \rightarrow V^{\otimes 2}$ of the rank $p$.

All even Hecke symmetries of rank 2 are classified (cf [4] and references therein).

Example 3. If $p=3$, then $\mathcal{P}_{-}(t)=1+n t+n t^{2}+t^{3}$.

Example 4. If $p=4$, then $\mathcal{P}_{-}(t)=1+n t+r t^{2}+n t^{3}+t^{4}$.

Definition 2.8. Let $\mathcal{P}_{-}(t)$ be a rational function represented as a ratio of two coprime polynomials, the degrees of the numerator and denominator being $p$ and $r$ respectively. We shall say that the corresponding Hecke symmetry has the bi-rank $(p \mid r)$.

The bi-rank of a super-flip coincides with its super-dimension.

Theorem 2.9. [10] Any Hecke symmetry has a certain bi-rank. In other words, its HP series $\mathcal{P}_{-}(t)$ is a rational function. 
Definition 2.10. A braiding $R$ is called skew-invertible if there exists an operator $\Psi: V^{\otimes 2} \rightarrow$ $V^{\otimes 2}$ such that

$$
\operatorname{Tr}_{(2)} R_{12} \Psi_{23}=P_{13}
$$

where $P_{13}$ is the flip transposing the first and the third spaces.

On fixing a basis $\left\{x_{i}\right\} \in V$ we get the corresponding basis $\left\{x_{i} \otimes x_{j}\right\}$ in the space $V^{\otimes 2}$. With respect to this basis the braiding $R$ and the operator $\Psi$ are represented by their matrices

$$
R\left(x_{i} \otimes x_{j}\right):=x_{k} \otimes x_{l} R_{i j}^{k l}, \quad \Psi\left(x_{i} \otimes x_{j}\right):=x_{k} \otimes x_{l} \Psi_{i j}^{k l}
$$

where the upper pair of indices enumerates columns of the matrix and the lower one - rows. In terms of matrices the relation in Definition 2.10 takes the form

$$
\sum_{a, b=1}^{n} R_{i a}^{k b} \Psi_{b j}^{a l}=\delta_{i}^{l} \delta_{j}^{k}
$$

Theorem 2.11 ([2]). If $R$ is a skew-invertible symmetry then the numerator $N(t)$ of the rational function $\mathcal{P}_{-}(t)$ is a reciprocal polynomial, while the denominator $D(t)$ is a skew-reciprocal one (i.e $D(-t)$ is reciprocal).

For any skew-invertible braiding the operators

$$
B:=\operatorname{Tr}_{(1)} \Psi \quad\left(B_{j}^{i}=\Psi_{a j}^{a i}\right), \quad C:=\operatorname{Tr}_{(2)} \Psi \quad\left(C_{j}^{i}=\Psi_{j a}^{i a}\right)
$$

are well defined. They are analogs of the parity operators in super-spaces. They are used for the definition of $R$-traces associated with $R$ in the spaces of endomorphisms. If End $(\mathrm{V})=\mathrm{V} \otimes \mathrm{V}^{*}$ is the space of left endomorphisms and the set $\left\{h_{i}^{j}\right\}$ is its natural basis (i.e $h_{i}^{j}\left(x_{k}\right)=\delta_{k}^{j} x_{i}$ ), then $\operatorname{Tr}_{R}\left(h_{i}^{j}\right)=C_{i}^{j}$ (for the space of right endomorphisms there is a similar formula with $B$ instead of $C$ ). Note that if $R$ is a super-flip then $\operatorname{Tr}_{R}$ is nothing but the usual super-trace.

\section{Quantum matrix algebras}

The most famous quantum matrix algebra related to a Hecke symmetry $R$ is the RTT-algebra. It is defined in following way. Let $T=\left\|t_{i}^{j}\right\|, 1 \leq i, j \leq n=\operatorname{dim} V$ be a matrix with entries $t_{i}^{j}$. Then by definition an RTT algebra is generated by the unit and by the elements $t_{i}^{j}$ subject to the relations

$$
R T_{1} T_{2}=T_{1} T_{2} R, \quad \text { where } \quad T_{1}:=T \otimes I, \quad T_{2}:=I \otimes T
$$

or in a coordinate form

$$
R_{i j}^{k l} t_{k}^{m} t_{l}^{n}=t_{i}^{k} t_{j}^{l} R_{k l}^{m n}
$$

where the summation over repeated indices is always understood. Being equipped with the coproduct

$$
\Delta\left(t_{i}^{j}\right)=t_{i}^{k} \otimes t_{k}^{j}
$$

and a counit it becomes a bi-algebra. If in addition $R$ is an even skew-invertible Hecke symmetry a group-like element $\operatorname{det}_{q}(T)$ (called the quantum determinant) can be defined. If it is central, the quotient of the RTT algebra over the ideal generated by $\operatorname{det}_{q}(T)-1$ is a Hopf algebra. For the standard Hecke symmetry ("the standard case" in what follows) this quotient is denoted 
$\mathbb{K}_{q}[S L(n)]$ and treated to be a quantum analog of the function space on the group $S L(n)$. Its restricted dual is just the quantum group $(\mathrm{QG}) U_{q}(s l(n))$.

Another important quantum matrix algebra associated with $R$ is the so-called Reflection Equation Algebra (REA). It is defined as follows. Let $L=\left\|l_{i}^{j}\right\|, 1 \leq i, j \leq n=\operatorname{dim} V$ be a matrix with entries $l_{i}^{j}$ (the lower index enumerates rows). Then the REA is generated by the unit and by the elements $l_{i}^{j}$ subject to the relations

$$
R L_{1} R L_{1}-L_{1} R L_{1} R=0
$$

or, in coordinates

$$
R_{i_{1} i_{2}}^{a_{1} b_{2}} l_{a_{1}}^{b_{1}} R_{b_{1} b_{2}}^{c_{1} j_{2}} l_{c_{1}}^{j_{1}}-l_{i_{1}}^{a_{1}} R_{a_{1} i_{2}}^{b_{1} c_{2}} l_{b_{1}}^{c_{1}} R_{c_{1} c_{2}}^{j_{1} j_{2}}=0
$$

This algebra has a braided bi-algebra structure and can be equipped with the coaction of the RTT algebra (in the standard case it can be also equipped with an action of the QG $\left.U_{q}(s l(n))\right)$. The term "braided" means that

$$
\Delta(a b)=\Delta(a) \Delta(b)=\left(a_{1} \otimes a_{2}\right)\left(b_{1} \otimes b_{2}\right):=a_{1} \tilde{b}_{1} \otimes \tilde{a}_{2} b_{2}
$$

where $\Delta(a)=a_{1} \otimes a_{2}, \tilde{d} \otimes \tilde{c}=R^{\operatorname{End}(\mathrm{V})}(c \otimes d)$, and $R^{\mathrm{End}(\mathrm{V})}$ is an extension of the initial Hecke symmetry $R$ to the REA (see the next section and [7] for more detail).

If $R$ is a skew-invertible even Hecke symmetry a group-like element $\overline{\operatorname{det}_{q}(L)}$ can be defined. Then the quotient of the REA over the ideal generated by $\overline{\operatorname{det}_{q}(L)}-1$ has a braided Hopf structure [15]. It is a braided analog of function space on $S L(n)$. Denote it $\overline{\mathbb{K}_{q}[S L(n)]}$.

In the standard case $\mathbb{K}_{q}[S L(n)]$ and $\overline{\mathbb{K}_{q}[S L(n)]}$ arise from the quantization of two different Poisson structures on the group $S L(n)$. The algebra $\mathbb{K}_{q}[S L(n)]$ arises from the Sklyanin bracket, while $\overline{\mathbb{K}_{q}[S L(n)]}$ originates from the Semenov-Tian-Shansky (S-T-S) one. A universal description for both these algebras is given in [11,5]. It is based on the use of a pair of compatible braidings, one of them being the Hecke symmetry.

However, the properties of $\mathbb{K}_{q}[S L(n)]$ and $\overline{\mathbb{K}_{q}[S L(n)]}$ differ drastically.

1. In the REA the elements ${ }^{3} \operatorname{Tr}_{R} L^{k}:=\operatorname{Tr}\left(C \cdot L^{k}\right)$ are central for any integer $k \geq 0$. In the RTT algebra their analogs are not central bur form a commutative subalgebra (i.e they are in involution).

2. For the REA there is a Cayley-Hamilton identity in the classical form (see section 5). For the RTT algebra the "powers" of the matrix $T$ coming in such a relation are defined in a more complicated way.

3. The REA has a further deformation which plays the role of the enveloping algebra of a "braided Lie algebra". We call this algebra the modified REA (mREA).

Definition 3.1. The mREA is an associative unital algebra, generated by the elements $l_{i}^{j}$ subject to the following quadratic-linear relations

$$
R L_{1} R L_{1}-L_{1} R L_{1} R=\hbar\left(R L_{1}-L_{1} R\right), \quad \hbar \in \mathbb{K} \backslash 0, \quad L:=\left\|l_{i}^{j}\right\|
$$

or, in components,

$$
R_{i_{1} i_{2}}^{a_{1} b_{2}} l_{a_{1}}^{b_{1}} R_{b_{1} b_{2}}^{c_{1} j_{2}} l_{c_{1}}^{j_{1}}-l_{i_{1}}^{a_{1}} R_{a_{1} i_{2}}^{b_{1} c_{2}} l_{b_{1}}^{c_{1}} R_{c_{1} c_{2}}^{j_{1} j_{2}}=\hbar\left(R_{i_{1} i_{2}}^{j_{1} a} l_{a}^{j_{2}}-l_{i_{1}}^{a} R_{a i_{2}}^{j_{1} j_{2}}\right)
$$

\footnotetext{
${ }^{3}$ Emphasize that the notation $\operatorname{Tr}_{R}$ plays a double role. From one side, it stands for the $R$-trace applied to the matrix $L$ or its powers. From the other side, when it is applied to an element of the space End(V) it sends this element to the field $\mathbb{K}$ as explained above.
} 
Theorem 3.2 ([7]). Let $R=R_{q}$ be a Hecke symmetry depending on $q \in \mathbb{K}$ (for $q=1$ it becomes involutive). Then for a generic $q$ the dimensions of homogeneous components of the RTT algebra and of the REA equal to those at $q=1$, that is the dimensions are stable under the q-deformation.

Moreover, there exists an analog of the PBW theorem for the mREA. Thus, in the standard case the mREA is a deformation algebra depending on 2 parameters $\hbar$ and $q$. Its Poisson counterpart is a Poisson pencil generated by the linear Poisson-Lie bracket on $g l(n)^{*}$ and by a quadratic bracket which is an extension of the S-T-S one to the whole space $g l(n)^{*}$. We refer the reader to the paper [7] for description of this Poisson pencil and to [16] for details on S-T-S bracket on the group.

In the sequel we use the notation $\mathcal{L}(q, \hbar)$ for the mREA and $\mathcal{L}(q)$ for the REA.

Note that the element $l=\operatorname{Tr}_{R} L:=C_{i}^{j} l_{j}^{i}$ is central in the both algebras $\mathcal{L}(q, \hbar)$ and $\mathcal{L}(q)$. So, it is natural to introduce the quotients $\mathcal{S} \mathcal{L}(q, \hbar)=\mathcal{L}(q, \hbar) /\langle l\rangle$ and $\mathcal{S} \mathcal{L}(q)=\mathcal{L}(q) /\langle l\rangle$ over an ideal, generated by $l$.

In the standard case all these algebras can be endowed with an action of the QG $U_{q}(\operatorname{sl}(n))$ so that

$$
X(a b)=X_{1}(a) X_{2}(b), \quad a, b \in \mathcal{L}(q) \text { or } \mathcal{L}(q, \hbar), \quad X \in U_{q}(s l(n)), \quad \Delta(X)=X_{1} \otimes X_{2}
$$

All the operators possessing this property are called equivariant.

\section{4 mREA and braided Lie bracket}

Let $R$ be a skew-invertible braiding. We want to equip the space $\operatorname{End}(\mathrm{V})$ (say, the left endomorphisms for the definiteness) with a structure of a generalized Lie algebra.

Let us first assume $R$ to be involutive $\left(R^{2}=1\right)$. Then there exists an extension of $R$ up to

$$
R^{\operatorname{End}(\mathrm{V})}: \operatorname{End}(\mathrm{V})^{\otimes 2} \rightarrow \operatorname{End}(\mathrm{V})^{\otimes 2}
$$

such that it is involutive and coordinated with the natural product

$$
\mu: \operatorname{End}(\mathrm{V})^{\otimes 2} \rightarrow \operatorname{End}(\mathrm{V})
$$

as follows

$$
R^{\mathrm{End}(\mathrm{V})} \mu_{23}=\mu_{12} R_{23}^{\mathrm{End}(\mathrm{V})} R_{12}^{\mathrm{End}(\mathrm{V})}
$$

Here the both sides of the equality should be applied to an element from End $(\mathrm{V})^{\otimes 3}$. This means that the result of applying the product $\mu$ does not depend on the position. The operators satisfying this property will be called $R$-invariant.

Let us set by definition

$$
[,]:=\mu\left(I-R^{\operatorname{End}(\mathrm{V})}\right)
$$

Theorem 4.1. The following properties hold true:

1. $R^{\operatorname{End}(\mathrm{V})}[,]_{23}=[,]_{12} R_{23}^{\operatorname{End}(\mathrm{V})} R_{12}^{\mathrm{End}(\mathrm{V})} \quad$ (the $R$-invariance of the bracket);

2. $[,] R^{\operatorname{End}(\mathrm{V})}=-[$,$] \quad (the R-skew-symmetry of the bracket);$

3. [, ] [, $]_{12}\left(I+R_{23}^{\operatorname{End}(\mathrm{V})} R_{12}^{\operatorname{End}(\mathrm{V})}+R_{12}^{\operatorname{End}(\mathrm{V})} R_{23}^{\mathrm{End}(\mathrm{V})}\right)=0 \quad$ (the R-Jacobi identity). 
Definition 4.2. A generalized Lie algebra, or $R$-Lie algebra, is a data

$$
\left(V, R: V^{\otimes 2} \rightarrow V^{\otimes 2},[,]: V^{\otimes 2} \rightarrow V\right)
$$

satisfying the properties $1-3$ above where $R^{\operatorname{End}(\mathrm{V})}$ should be replaced by $R$.

The generalized Lie algebra defined above in the space $\operatorname{End}(\mathrm{V})$ is denoted $g l\left(V_{R}\right)$.

Let $\operatorname{Tr}_{R}: \operatorname{End}(\mathrm{V}) \rightarrow \mathbb{K}$ be the $R$-trace associated with a given skew-invertible involutive symmetry $R$ (see section 2). The family of $\operatorname{Tr}_{R}$-less elements of $g l\left(V_{R}\right)$ forms a generalized Lie subalgebra denoted $s l\left(V_{R}\right)$. For any generalized Lie algebra $\mathfrak{g}$ its enveloping algebra can be defined in the natural way as the following quotient

$$
U(\mathfrak{g})=T(\mathfrak{g})) /\langle\operatorname{Im}(\mathrm{I}-\mathrm{R}-[,])\rangle
$$

It is a braided Hopf algebra, its coproduct being additive on the generators:

$$
\Delta(X)=X \otimes 1+1 \otimes X, \quad \forall X \in \mathfrak{g} .
$$

We would like to extend this construction to the non-involutive case. However, in contrast to the involutive case, here we first define the "enveloping algebra" of a braided Lie bracket, and then the bracket itself. We consider the mREA $\mathcal{L}(q, \hbar)$ corresponding to a skew-invertible Hecke symmetry $R$ as a proper analog of the enveloping algebra $U(g l(n))$.

In order to argue this point of view we restrict ourselves to the standard Hecke symmetries. The following statement is a corollary of the theorem 3.2.

Proposition 4.3. The mREA corresponding to a standard Hecke symmetry is a two-parameter deformation of the commutative algebra $\mathrm{Sym}(\mathrm{gl}(\mathrm{n}))$ and a one-parameter deformation of $U\left(g l(n)_{\hbar}\right)$ (the subscript $\hbar$ means that the parameter $\hbar$ stands as a multiplier at the usual $g l(n)$ Lie bracket).

Any finite dimensional representation of $U(g l(n))$ can be deformed into an equivariant representation of the $m R E A \mathcal{L}(q, \hbar)$.

Besides, a sort of the PBW theorem is valid for the mREA (cf [7]). Concerning simple algebras of the series $B_{n}, C_{n}, D_{n}$ there exists no similar deformation of their enveloping algebras.

Now, we are able to define a braided analog of the Lie bracket arising from $\mathcal{L}(q, \hbar)$. Below we put $\hbar=1$. The commutative relations among the generators $l_{i}^{j}$ can be rewritten as follows $(\operatorname{cf}[7])$ :

$$
l_{i}^{j} l_{k}^{l}-Q\left(l_{i}^{j} l_{k}^{l}\right)=\text { linear terms }
$$

Denoting the right hand side of the above relation as $\left[l_{i}^{j}, l_{k}^{l}\right]$ we claim the following.

Theorem 4.4. Consider the map $\mathcal{L}(q, 1) \rightarrow \operatorname{End}(\mathcal{L}(q, 1)): l_{i}^{j} \mapsto L_{i}^{j}$ where $L_{i}^{j}$ is a linear operator acting on generators by the rule $L_{i}^{j}\left(l_{k}^{l}\right):=\left[l_{i}^{j}, l_{k}^{l}\right]$. This map is a representation of the algebra $\mathcal{L}(q, 1)$.

We shall call the map $l_{i}^{j} \mapsto L_{i}^{j}$ the adjoint representation, $L_{i}^{j}$ the adjoint operator and the operation [, ] the braided Lie bracket. Also, we denote the vector space $\operatorname{Span}\left(l_{i}^{j}\right)$ endowed with the braided Lie bracket as $g l\left(V_{R}\right)$. Unfortunately, the properties listed in the theorem 4.1 (where $R$ is assumed to be an involutive symmetry) are not valid in general case. Their analogs for the braided Lie bracket in question are more complicated (cf $[7,9]$ for detail).

In fact, we have identified the space $\operatorname{Span}\left(l_{i}^{j}\right)$ with $\operatorname{End}(\mathrm{V})$. The $R$-trace in the basis $\left\{l_{i}^{j}\right\} \in$ $\operatorname{End}(\mathrm{V})$ is $\operatorname{Tr}_{R} l_{i}^{j} \sim \delta_{i}^{j}$ (up to a factor). It is not difficult to see that traceless elements of the 
braided Lie algebra $g l\left(V_{R}\right)$ form a subalgebra $s l\left(V_{R}\right)$. Moreover, if $\operatorname{Tr} C \neq 0$ there exists a natural projector $g l\left(V_{R}\right) \rightarrow s l\left(V_{R}\right)$ similar to the classical one.

However, the restriction of the adjoint representation of the algebra $g l\left(V_{R}\right)$ to the subalgebra $s l\left(V_{R}\right)$ is not in general a representation of the latter algebra. However, by a slight modification of this restriction (described in [7], section 6) we can get an analog of the adjoint representation of the algebra $s l(n)$.

Note that in the standard case the braided analogs of the $g l(n)$ and $\operatorname{sl}(n)$ adjoint representations are equivariant. Also, note that by using the methods of [14] a map from $\mathcal{S} \mathcal{L}(q, 1)$ to the QG $U_{q}(s l(n))$ (localized by the quantum Casimir element) can be constructed. Basing on this map it is possible to develop a representation theory of the algebra $\mathcal{S} \mathcal{L}(q, 1)$ in the standard case.

\section{Cayley-Hamilton identity}

Let $R$ be a skew-invertible Hecke symmetry and $\lambda$ be a partition. Let us consider the corresponding REA or mREA and define a Schur function $s_{\lambda}(L)$ as follows

$$
s_{0}=1, \quad s_{\lambda}(L):=\left(\operatorname{Tr}_{R}\right)_{1 \ldots k}\left(L_{\overline{1}} \ldots L_{\bar{k}} \rho_{R}\left(E_{\alpha}^{\lambda}\right)\right), \quad \lambda \vdash k, \quad k=1,2, \ldots
$$

Here

$$
L_{\overline{1}}=L, \quad L_{\overline{k+1}}=R_{k k+1} L_{\bar{k}} R_{k k+1}^{-1}
$$

$E_{\alpha}^{\lambda}$ is a primitive idempotent of the Hecke algebra $H_{n}(q)$ corresponding to a partition $\lambda$. The symbol $\rho_{R}$ stands for the "local" representation of $H_{n}(q)$ associating the braidings $R_{k k+1}$ with the standard generators $\sigma_{k} \in H_{n}(q)$.

Theorem 5.1. The elements $s_{\lambda}(L)$ are central in the algebra $\mathcal{L}(q)$.

Theorem 5.2. [Cayley-Hamilton theorem] Assume that $R$ is an even Hecke symmetry of rank $p$. Then the matrix $L=\left\|l_{i}^{j}\right\|$ composed of the REA generators satisfies the following CayleyHamilton (CH) identity

$$
L^{p}-q s_{(1)}(L) L^{p-1}+q^{2} s_{\left(1^{2}\right)}(L) L^{p-2}-\cdots+(-q)^{p} s_{\left(1^{p}\right)}(L) I=0
$$

Here the partition $\left(1^{k}\right)$ is represented by the one-column Young diagram of the height $k$.

Remark 5.3. In fact, this $\mathrm{CH}$ identity is the first one in a family of $\mathrm{CH}$ identities. It is called the basic identity. The other identities of the family deals with some extensions of the basic matrix $L$ and they are called the higher identities.

Let us introduce formal elements $\mu_{1}, \mu_{2}, \ldots, \mu_{p}$ such that

$$
q^{k} s_{\left(1^{k}\right)}(L)=\sum_{1 \leq i_{1}<\cdots<i_{k} \leq p} \mu_{i_{1}} \ldots \mu_{i_{k}}
$$

Otherwise stated, $\mu_{i}$ are the roots of the equation

$$
\mu^{p}-q s_{(1)}(L) \mu^{p-1}+q^{2} s_{\left(1^{2}\right)}(L) \mu^{p-2}-\cdots+(-q)^{p} s_{\left(1^{p}\right)}(L) I=0
$$

So, $\mu_{i}$ belong to an algebraic extension of the center of the algebra $\mathcal{L}(q)$. Then the $\mathrm{CH}$ identity factorizes into the product

$$
\prod_{i=1}^{p}\left(L-\mu_{i}\right)=0
$$


Let us fix values of the elements $\mu_{i}$ and let $\mathcal{L}^{\mu}(q)$ denotes the quotient of the REA over the ideal generated by the set of elements

$$
\left\{q^{k} s_{\left(1^{k}\right)}(L)-\sum_{1 \leq i_{1}<\cdots<i_{k} \leq p} \mu_{i_{1}} \ldots \mu_{i_{k}} \mid 1 \leq k \leq p\right\}
$$

We assume the family $\left\{\mu_{1}, \ldots, \mu_{p}\right\}$ to be generic (in particular, $\mu_{i}$ are pairwise distinct). Then the algebra $\mathcal{L}^{\mu}(q)$ is a braided analog of the coordinate algebra of a generic coadjoint orbit in $g l(n)^{*}$. This means that in the standard case this algebra arises from the quantization of a generic orbit in $g l(n)^{*}$.

Proposition 5.4. The elements

$$
e_{i}(L)=\prod_{j \neq i} \frac{L-\mu_{j}}{\mu_{i}-\mu_{j}}
$$

are pairwise orthogonal idempotents (projectors)

$$
e_{i}(L)^{2}=e_{i}(L), \quad e_{i}(L) e_{j}(L)=0, i \neq j, \quad \sum_{i=1}^{p} e_{i}(L)=I
$$

Recall that the elements $\operatorname{Tr}_{R} L^{k}$ are central in the algebra $\mathcal{L}(q)$.

Theorem 5.5. In the algebra $\mathcal{L}^{\mu}(q)$ there is a spectral decomposition

$$
\operatorname{Tr}_{R} L^{k}=\sum_{i=1}^{p} \mu_{i}^{k} d_{i}, \quad \text { where } \quad d_{i}=\operatorname{Tr}_{R} e_{i}(L)=\prod_{j \neq i} \frac{q \mu_{i}-q^{-1} \mu_{j}}{\mu_{i}-\mu_{j}}
$$

Here $\operatorname{Tr}_{R}$ is normalized by he condition $\operatorname{Tr}_{R} I=p_{q}=\frac{q^{p}-q^{-p}}{q-q^{-1}}$. The quantities $d_{i}$ are called the quantum dimensions.

Using the idempotents $e_{i}(L)$ we can define quantum analogs of line bundles over the quantum orbits via projective modules in the spirit of the Serre-Swan's approach.

Note that a $\mathrm{CH}$ identity is also valid for the algebra $\mathcal{L}(q, \hbar)$. For this algebra the formula similar to (5.1) takes place too, but $\mu_{i}$ become now the roots of the $\mathrm{CH}$ identity for $\mathcal{L}(q, \hbar)$ and the quantum dimensions should be modified as follows

$$
d_{i}=\operatorname{Tr}_{R} e_{i}(L)=\prod_{j \neq i} \frac{q \mu_{i}-q^{-1} \mu_{j}-\hbar}{\mu_{i}-\mu_{j}}
$$

In order to get the latter formula it suffices to replace the roots $\mu_{i}$ in $(5.1)$ by $\mu_{i}-\frac{\hbar}{q-q^{-1}}$.

Formula (5.1) can be also considered as a parameterized relation between two families of central elements, namely, $\left\{\operatorname{Tr}_{R} L^{k}\right\}, 1 \leq k \leq p$, and the set of coefficients of the corresponding $\mathrm{CH}$ identity in the algebras $\mathcal{L}(q)$ or $\mathcal{L}(q, \hbar)$.

Now let us pass to the Hecke symmetries of $G L(p \mid r)$ type.

Theorem 5.6 ([5]). Let now $R$ be a skew-invertible Hecke symmetry of the bi-rank $(p \mid r)$. The matrix $L=\left\|l_{i}^{j}\right\|$ composed of the REA generators satisfies the following CH identity

$$
\sum_{i=0}^{p+r} L^{p+r-i} \sum_{k=\max \{0, i-r\}}^{\min \{i, p\}}(-1)^{k} q^{2 k-i} s_{[p \mid r]_{i-k}^{k}}(L)=0
$$


where $[p \mid r]_{i-k}^{k}$ denotes the partition with the following Young diagram

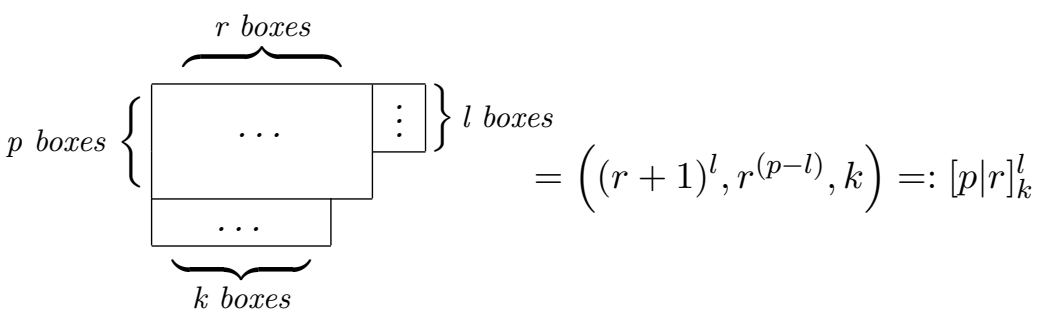

This is a $q$-generalization of the Kantor-Trishin's result [13].

Theorem $5.7([6])$. Being multiplied by $s_{[p \mid r]}$, the CH of Theorem 5.6 factorizes into the product

$$
\left(\sum_{k=0}^{p}(-q)^{k} L^{p-k} s_{[p \mid r]^{k}}(L)\right)\left(\sum_{l=0}^{r} q^{-l} L^{r-l} s_{[p \mid r]_{l}}(L)\right)=0
$$

Denoting the roots of the first and of the second factors as $\left\{\mu_{i}\right\}_{1 \leq i \leq p}$ and $\left\{\nu_{j}\right\}_{1 \leq j \leq r}$ respectively, we get the following parameterizations of the normalized coefficients of these factors

$$
\begin{aligned}
& \frac{s_{[p \mid r]^{k}(L)}}{s_{[p \mid r](L)}} \mapsto \frac{s_{[p \mid r]}(\mu, \nu)}{s_{[p \mid r]}(\mu, \nu)}:=\sum_{1 \leq i_{1}<\cdots<i_{p} \leq p} \quad q^{-k} \mu_{i_{1}} \ldots \mu_{i_{k}}=e_{k}\left(q^{-1} \mu\right), \quad 1 \leq k \leq p \\
& \frac{s_{[p \mid r]_{l}}(L)}{s_{[p \mid r]}(L)} \mapsto \frac{s_{[p \mid r]_{l}}(\mu, \nu)}{s_{[p \mid r]}(\mu, \nu)}:=\sum_{1 \leq j_{1}<\cdots<j_{l} \leq r}(-q)^{l} \nu_{j_{1}} \ldots \nu_{j_{l}}=e_{l}(-q \nu), \quad 1 \leq l \leq r
\end{aligned}
$$

In terms of "even" roots $\mu_{i}$ and "odd" roots $\nu_{j}$ the $\mathrm{CH}$ identity factorizes as follows

$$
\left(s_{[p \mid r]}(L)\right)^{2} \prod_{i=1}^{p}\left(L-\mu_{i} I\right) \prod_{j=1}^{r}\left(L-\nu_{j} I\right)=0
$$

Recently a formula similar to (5.1) has been obtained (the proof will be given in our forthcoming paper).

Theorem 5.8. Let $\mathcal{L}(q)^{\mu, \nu}$ be the quotient algebra of the $G L(p \mid r)$ type $R E A$ by the relations (5.2), (5.3). In $\mathcal{L}(q)^{\mu, \nu}$ the following relations hold true

$$
\operatorname{Tr}_{R} L^{k}=\sum_{i=1}^{p} d_{i} \mu_{i}^{k}+\sum_{j=1}^{r} \widetilde{d}_{j} \nu_{j}^{k}
$$

where

$$
\begin{aligned}
& d_{i}:=q^{-1} \prod_{s=1, s \neq i}^{p} \frac{\mu_{i}-q^{-2} \mu_{s}}{\mu_{i}-\mu_{s}} \prod_{j=1}^{r} \frac{\mu_{i}-q^{2} \nu_{j}}{\mu_{i}-\nu_{j}} \\
& \widetilde{d}_{j}:=-q \prod_{i=1}^{p} \frac{\nu_{j}-q^{-2} \mu_{i}}{\nu_{j}-\mu_{i}} \prod_{s=1, s \neq j}^{r} \frac{\nu_{j}-q^{2} \nu_{s}}{\nu_{j}-\nu_{s}}
\end{aligned}
$$

Similarly to the even case, if we replace $\mu_{i}$ (resp., $\nu_{j}$ ) by $\mu_{i}-\frac{\hbar}{q-q^{-1}}$ (resp., $\nu_{j}-\frac{\hbar}{q-q^{-1}}$ ) we get the "quantum dimensions" $d_{i}$ (resp., $\widetilde{d}_{j}$ ) valid for the spectral decomposition in the algebra $\mathcal{L}(q, \hbar)$. In the modified formula $\mu_{i}$ and $\nu_{j}$ are regarded to be the roots of the $\mathrm{CH}$ identity for the matrix $L$ composed of the generators of the algebra $\mathcal{L}(q, \hbar)$. 


\section{Example: $q$-Minkowski space and $q$-hyperboloid algebras}

Let $R$ be the standard Hecke symmetry in the case $n=2$. In an appropriate basis $\{x, y\}$ of the two dimensional space $V$ we get the following matrix

$$
R=\left(\begin{array}{cccc}
q & 0 & 0 & 0 \\
0 & q-q^{-1} & 1 & 0 \\
0 & 1 & 0 & 0 \\
0 & 0 & 0 & q
\end{array}\right)
$$

which represents this Hecke symmetry in the basis $\{x \otimes x, x \otimes y, y \otimes x, y \otimes y\}$.

Also, we put $L=\left(\begin{array}{ll}l_{1}^{1} & l_{1}^{2} \\ l_{2}^{1} & l_{2}^{2}\end{array}\right)=\left(\begin{array}{cc}a & b \\ c & d\end{array}\right)$. Then the system defining the mREA becomes

$$
\begin{array}{ll}
q a b-q^{-1} b a=\hbar b & q(b c-c b)=\left(\left(q-q^{-1}\right) a-\hbar\right)(d-a) \\
q c a-q^{-1} a c=\hbar c & q(c d-d c)=c\left(\left(q-q^{-1}\right) a-\hbar\right) \\
a d-d a=0 & q(d b-b d)=\left(\left(q-q^{-1}\right) a-\hbar\right) b
\end{array}
$$

If $\hbar=0$ this algebra is called the $q$-Minkowski space algebra [12]. For $\hbar \neq 0$ it is regarded to be a braided or $q$-analog of the enveloping algebra $U\left(g l(2)_{\hbar}\right)$.

Changing the set of generators $\{a, d, b, c\}$ for $\{l, h, b, c\}$ where

$$
l=q^{-1} a+q d, \quad h=a-d
$$

we come to the relations

$$
\begin{array}{ll}
q^{2} h b-b h=\hbar 2_{q} b-\left(q-q^{-1}\right) l b, & b l=l b \\
q^{2} c h-h c=\hbar 2_{q} c-\left(q-q^{-1}\right) l c, & c l=l c \\
\left(q^{2}+1\right)(b c-c b)+\left(q^{2}-1\right) h^{2}=\hbar 2_{q} h-\left(q-q^{-1}\right) l h, & h l=l h
\end{array}
$$

In this basis of generators it is seen explicitly that the element $l=q^{-1} a+q d$ is central. Therefore, we can introduce the quotient algebra

$$
\mathcal{S} \mathcal{L}(q, \hbar)=\mathcal{L}(q, \hbar) /\langle l\rangle
$$

The commutation relations among the independent generators of $\mathcal{S} \mathcal{L}(q, \hbar)$ read

$$
q^{2} h b-b h=\hbar 2_{q} b, \quad q^{2} c h-h c=\hbar 2_{q} c, \quad\left(q^{2}+1\right)(b c-c b)+\left(q^{2}-1\right) h^{2}=\hbar 2_{q} h
$$

Thus, we get a braided or $q$-counterpart of the algebra $U\left(\operatorname{sl}(2)_{\hbar}\right)$. But in contrast with the classical case, the algebra $\mathcal{S} \mathcal{L}(q, \hbar)$ is not a subalgebra of $\mathcal{L}(q, \hbar)$.

Consider now the central element $\operatorname{Tr}_{q} L^{2} \in \mathcal{L}(q, \hbar)$. Its explicit form in the basis $\{l, h, b, c\}$ reads

$$
\operatorname{Tr}_{q} L^{2}=q^{-1} b c+q c b+\frac{q}{1+q^{2}}\left(h^{2}+l^{2}\right)
$$

Its image $C$ in the algebra $\mathcal{S} \mathcal{L}(q, \hbar)$ is

$$
C=q^{-1} b c+q c b+\frac{q}{1+q^{2}} h^{2} \in \mathcal{S} \mathcal{L}(q, \hbar)
$$

This element is central in the algebra $\mathcal{S} \mathcal{L}(q, \hbar)$. The quotient of this algebra over the ideal $\langle C-\alpha\rangle, \alpha \neq 0$, is called the quantum (or braided or $q$-)hyperboloid algebra

$$
\mathcal{A}_{\alpha}:=\mathcal{S} \mathcal{L}(q, \hbar) /\langle C-\alpha\rangle
$$


On the next step we introduce the braided Lie algebra $s l\left(V_{R}\right)$. But in the low-dimensional case in question we can simplify the construction of the corresponding braided Lie bracket. We only use the fact that this bracket is $U_{q}(s l(2))$-covariant. We put $\mathcal{S} \mathcal{L}=\operatorname{Span}(b, h, c)$. Let us equip this space with an action of the QG $U_{q}(s l(2))$ and extend this action to the space $\mathcal{S L} \otimes \mathcal{S} \mathcal{L}$ by using the coproduct of the QG. Then for a generic $q$ the space $\mathcal{S L} \otimes \mathcal{S L}$ can be decomposed into a direct sum of irreducible $U_{q}(\operatorname{sl}(2))$ submodules $\mathcal{S} \mathcal{L} \otimes \mathcal{S} \mathcal{L}=V_{0} \oplus V_{1} \oplus V_{2}$ where the subscript stands for the spin. Then the operator

$$
[,]: \mathcal{S L} \otimes \mathcal{S L} \rightarrow \mathcal{S L}
$$

is a $U_{q}(s l(2))$ morphism iff it is trivial on the components $V_{0}$ and $V_{2}$ and is an isomorphism between $V_{1}$ and $\mathcal{S} \mathcal{L}$. By this property the bracket is uniquely defined up to a nonzero factor $w$.

Let us exhibit the multiplication table of this braided bracket:

$$
\begin{aligned}
& {[b, b]=0, \quad[b, h]=-w b, \quad[b, c]=w \frac{q}{2_{q}} h, \quad[h, b]=w q^{2} b} \\
& {[h, h]=w\left(q^{2}-1\right), \quad[h, c]=-w c, \quad[c, b]=-w \frac{q}{2_{q}} h, \quad[c, h]=w q^{2} c, \quad[c, c]=0}
\end{aligned}
$$

As can be easily seen, the bracket [, ] defines a representation of the algebra $\mathcal{S} \mathcal{L}(q, 1)$ iff $w=$ $\hbar\left(q^{4}-q^{2}+1\right)^{-1}$.

Consider now $q$-analogs of adjoint operators. Being represented by matrices in the basis $\{b, h, c\}$ they have the form

$$
B_{q}=w\left(\begin{array}{ccc}
0 & -1 & 0 \\
0 & 0 & \frac{q}{2_{q}} \\
0 & 0 & 0
\end{array}\right), \quad H_{q}=w\left(\begin{array}{ccc}
q^{2} & 0 & 0 \\
0 & q^{2}-1 & 0 \\
0 & 0 & -1
\end{array}\right), \quad C_{q}=w\left(\begin{array}{ccc}
0 & 0 & 0 \\
-\frac{q}{2_{q}} & 0 & 0 \\
0 & q^{2} & 0
\end{array}\right)
$$

Theorem 6.1. The operators $B_{q}, H_{q}, C_{q}$ satisfy the relation

$$
q^{-1} b C_{q}+\frac{h H_{q}}{2_{q}}+q c B_{q}=0
$$

Moreover, there exist extensions of these operators to the higher homogeneous components of the algebra $\mathcal{S L}(q)$ such that on each component we get a representation of the algebra $\mathcal{S} \mathcal{L}(q, 1)$ and this relation is still valid for the extended operators $B_{q}, H_{q}, C_{q}$ (we keep for them the same notations).

Now, we are able to define a braided analog of the space of vector fields on the classical hyperboloid. Let us consider the left $\mathcal{A}_{\alpha}$-module generated by the operators $B_{q}, H_{q}, C_{q}$. It is possible to show that this $\mathcal{A}_{\alpha}$-module is projective. It is natural to call it the "tangent module" on the $q$-hyperboloid in question. In a similar way the "cotangent module" on this $q$-hyperboloid can be introduced (it is isomorphic to the tangent one). Such braided geometrical structures on the quantum hyperboloid were first considered by one of the authors (D.G.) and P.Akueson (cf [1] and the references therein).

Note that the above vector fields are very useful for defining braided analogs of some operators of mathematical physics on the $q$-hyperboloid. Thus, the Laplace operator on a $q$-hyperboloid can be defined via the element (6.1) where the generators $b, h, c$ should be replaced by braided vector fields $B_{q}, H_{q}, C_{q}$ respectively. As a result, we get the following braided Laplace operator on the $q$-hyperboloid

$$
q^{-1} B_{q} C_{q}+\frac{H_{q}^{2}}{2_{q}}+q C_{q} B_{q}
$$


A braided version of the Dirac operator on the $q$-hyperboloid can be be defined a similar way. Recently a braided analog of the Maxwell operator on the $q$-hyperboloid was constructed. Its construction will be published in [3].

It would be very interesting to generalize these constructions and results on other "braided varieties" of general type. The most intriguing problem is whether the module of the braided vector fields defined on these "varieties" possesses the properties analogous to those considered above.

\section{Acknowledgement}

The work of D. G. was partially supported by the grant ANR-05-BLAN-0029-01, the work of P. P. and P. S. was partially supported by the RFBR grant 05-01-01086.

\section{References}

[1] P. Akueson. Géométrie de l'espace tangent sur l'hyperboloid quantique. Cahiers Topologie Géom. Différentielle Catég, 42 (2001), 2-50.

[2] N. P. Dung and P. H.Hai. On the Poincaré series of quadratic algebras associated to Hecke symmetries. Int. Math. Res. Notes, 40 (2003), 2193-2203.

[3] A. Dutriaux and D. Gurevich. Maxwell operator on $q$-Minkowski space and quantum sphere. J. Phys. A (submitted).

[4] D. Gurevich. Algebraic aspects of the Yang-Baxter equation. Translation in Leningrad Math. J. 2 (1991), 801-828.

[5] D. Gurevich, P. Pyatov, and P. Saponov. The Cayley-Hamilton theorem for quantum matrix algebras of $G L(m \mid n)$ type. Translation in St. Petersburg Math. J. 17 (2006), 119-135.

[6] D. Gurevich, P. Pyatov, and P. Saponov. Quantum matrix algebras of $G L(m \mid n)$ type: the structure of characteristic subalgebra and its parameterization. Teor. Mat. Fiz. 147 (2006), 14-46 (in Russian).

[7] D. Gurevich, P. Pyatov, and P. Saponov. Representation theory of (modified) Reflection Equation Algebra of $G L(m \mid n)$ type. Translation in St. Petersburg Math. J. (to be published).

[8] D. Gurevich and P. Saponov. Geometry of non-commutative orbits related to Hecke symmetries. in "Proceedings of the International Conference in memory of Joseph Donim held at the TechnionIsrael Institute of Technology, Haifa, July 5-12, 2004“. P. Etingof, S. Gelaki, and S. Shnider, Eds. Contem. Math. 433 (2007), 209-250.

[9] D. Gurevich and P. Saponov. Quantum Lie algebras via modified Reflection Equation Algebra. Proceedings of Satelite Conference "From Lie Algebras to Quantum Groups" (Coimbra 2006), Ed. CIM, 28 (2007), 107-124.

[10] P. H. Hai. Poincaré Series of quantum spaces associated to Hecke operators. Acta Math. Vietnam, 24 (1999), 235-246.

[11] A. Isaev, O. Ogievetsky, and P. Pyatov. On quantum matrix algebras satisfying the Cayley-HamiltonNewton identities. J. Phys. A: Math. Gen. 32 (1999), L115-L121.

[12] P. P. Kulish. Representations of $q$-Minkowski space algebra. St. Petersburg Math. J. 6 (1995) 365374; Alg. Anal. 6 (1994), 195-205; preprint arXiv:hep-th/9312139.

[13] I. Kantor and I. Trishin. On a concept of determinant in the supercase. Comm. Algebra, 27 (1999), 233-259.

[14] V. Lyubashenko and A. Sudbery. Generalized Lie algebras of type $A_{n}$. J. Math. Phys. 39 (1998), 3487-3504.

[15] S. Majid. Foundations of Quantum Group Theory. Cambridge University Press, Cambridge, 1995.

[16] A. Mudrov. On quantization of the Semenov-Tian-Shansky Poisson bracket on simple algebraic groups. Translation in St. Petersburg Math. J. 18 (2007), 797-808.

[17] N. Reshetikhin, L. Takhtajan, and L. Faddeev. Quantization of Lie groups and Lie algebras. Translation in Leningrad Math. J. 1 (1990), 193-225. 